\title{
Development of Standardized Lunar Regolith Simulant Materials
}

\author{
P. Carpenter ${ }^{*}$, L. Sibille* ${ }^{*}$ S. Wilson, ${ }^{* *}$ and G. Meeker ${ }^{* *}$ \\ *BAE Systems, Marshall Space Flight Center, AL 35812 \\ ** United States Geological Survey, MS964, Lakewood CO 80025
}

Lunar exploration requires scientific and engineering studies using standardized testing procedures that ultimately support flight certification of technologies and hardware. It is necessary to anticipate the range of source materials and environmental constraints that are expected on the Moon and Mars, and to evaluate in-situ resource utilization (ISRU) coupled with testing and development. We describe here the development of standardized lunar regolith simulant (SLRS) materials that are traceable inter-laboratory standards for testing and technology development. These SLRS materials must simulate the lunar regolith in terms of physical, chemical, and mineralogical properties. A summary of these issues is contained in the 2005 Workshop on Lunar Regolith Simulant Materials [1]. Lunar mare basalt simulants MLS-1 and JSC-1 were developed in the late 1980s. MLS-1 approximates an Apollo 11 high-Ti basalt, and was produced by milling of a holocrystalline, coarse-grained intrusive gabbro (Fig. 1). JSC1 approximates an Apollo 14 basalt with a relatively low-Ti content, and was obtained from a glassy volcanic ash (Fig. 2). Supplies of MLS-1 and JSC-1 have been exhausted and these materials are no longer available. No highland anorthosite simulant was previously developed. Upcoming lunar polar missions thus require the identification, assessment, and development of both mare and highland simulants.

A lunar regolith simulant is manufactured from terrestrial components for the purpose of simulating the physical and chemical properties of the lunar regolith. Significant challenges exist in the identification of appropriate terrestrial source materials. Lunar materials formed under comparatively reducing conditions in the absence of water, and were modified by meteorite impact events. Terrestrial materials formed under more oxidizing conditions with significantly greater access to water, and were modified by a wide range of weathering processes. The composition space of lunar materials can be modeled by mixing programs utilizing a low-Ti basalt, ilmenite, KREEP component, high-Ca anorthosite, and meteoritic components. This approach has been used for genetic studies of lunar samples via chemical and modal analysis. A reduced composition space may be appropriate for simulant development, but it is necessary to determine the controlling properties that affect the physical, chemical and mineralogical components of the simulant.

A root simulant represents an end-member in terms of simulant properties such as bulk chemistry, and a derivative simulant is formed from a root by modification or addition of material [2]. The fidelity of the simulant represents the degree of duplication of soil characteristics compared to lunar soils. The 2005 Workshop recommended production of two root simulants corresponding to a low-Ti mare basalt and a high-Ca highland anorthosite. These roots represent compositional end-members of mare and highland materials, and can in principle be physically mixed to target the range of soil compositions in the Apollo inventory. The low-Ti mare root under consideration is similar to an Apollo 15 low-Ti basalt, and compositions richer in Fe and $\mathrm{Ti}$ can be simulated by addition of ilmenite to this low-Ti root. Specific lunar regolith properties can be addressed by addition of ilmenite, glassy agglutinates, nanophase iron, and other materials [3].

An ideal SLRS material has high fidelity, is homogeneous, widely available, and inexpensive to produce and purchase. Fidelity and homogeneity are important, on the scale of material supplied to the end user, and on the scale of a production run. Homogeneity is ensured in geochemical standards (finely-ground rock powders) by reducing the grain size to accomplish reduced chemical variability. Small aliquots of the standard are representative of the master batch as each aliquot contains a large number of grains. Conversely, lunar simulants have a grain size variation and modal mineralogy at each size fraction that must be retained in order to match the target lunar regolith. As the sample size is reduced, at some point it is no longer representative. The deviation in properties can be monitored by chemical analysis using progressively smaller sample sizes, in comparison with analyses performed on bulk material. This problem has been observed in analyses of MLS- 1 , where a $\sim 10 \%$ variation in $\mathrm{SiO}_{2}$ is observed, compared to a $160 \%$ variation for $\mathrm{Cr}$ [1]. This chemical contrast illustrates small differences in silicate minerals for the major element $\mathrm{Si}$, but large differences in minerals for the trace element $\mathrm{Cr}$ since it is con- 
centrated in chromite. Similarly, geotechnical properties may be dominated by a large difference in mineral hardness, and rogue grains such as quartz could stand out in tests using too small a quantity of material. The variability of simulant material thus is an inherent property but must be taken into account for both quality control and for simulant use by the scientific community.

The workshop recommended redeployment of JSC-1 to serve as a testing material for immediate needs while root simulants are developed. Simulant JSC-1A, and a dust size fraction of JSC-1A (for studies on human toxicology and mechanical abrasion) are being produced under the direction of Marshall Space Flight Center (MSFC). Root simulant production requires: (1) establishment of simulant requirements, based on analysis of lunar regolith properties and planned activities on the lunar surface; (2) screening and selection of appropriate terrestrial source materials; (3) milling and other processing necessary to produce the required physical attributes such as grain size distribution; and (4) characterization of chemistry, mineralogy, and geotechnical properties in order to monitor the adherence to simulant requirements and homogeneity during production. Simulant is currently being characterized during critical stages of production and packaging, and this quality control includes traceability using geochemical reference standards. An efficient and comprehensive analytical program has been developed which includes bulk and microanalytical methods. Development of derivative simulants will include production of synthetic agglutinates using plasma fusion melting to produce glassy microspheres. These synthetic agglutinates may also be processed to contain nanophase iron inclusions and vesicles in order to increase the fidelity of the simulant. Ion implantation can be utilized to simulate exposure to the solar wind.

\section{References}

[1] Sibille L. et al., (2005) Lunar Regolith Simulant Materials: Recommendations for Standardization, Production, and Usage. NASA TP (In preparation, http://est.msfc.nasa.gov/workshops/lrsm2005.html)

[2] Carter J., et al. (2004) Space Resources Roundtable VI, 15.

[3] Taylor L. et al. (2004) Space Resources Roundtable VI, 46.
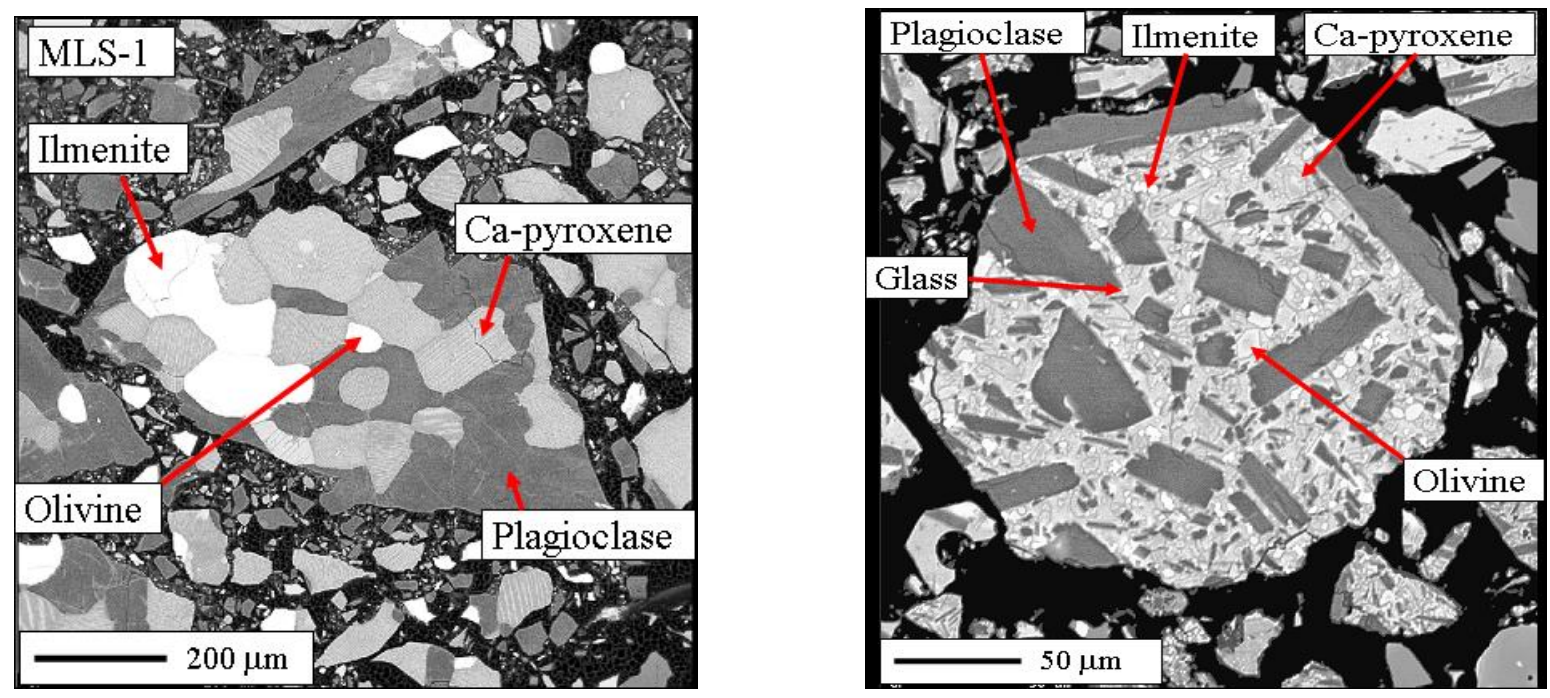

Fig, 1. (Left) Backscattered-electron (BSE) image of lunar simulant MLS-1 (polished mount) illustrating coarse grain size, and absence of glass. MLS-1 has monomineralic grains of the same size as lithic fragments in JSC-1. Compared to Apollo 11 soils, MLS-1 represents a good match for composition, but a poor match for texture.

Fig. 2. (Right) BSE image of lunar simulant JSC-1 (polished mount). Grain size distribution of JSC-1 is similar to Apollo soils. Glass content and fine grain size of JSC-1 is desirable in a mare lunar simulant, but no equivalent of lunar agglutinates is present and must be added to form a derivative simulant. 